\title{
Comparison between unimodal and multimodal Physical Therapy interventions in frailty: a systematic review
}

\section{Comparacão entre intervencões fisioterapêuticas unimodais e multimodais na Síndrome da Fragilidade: revisão sistemática}

Maria do Socorro Morais Pereira Simões ${ }^{1}$

Patrícia Albuquerque de Moura ${ }^{1}$

Renata dos Ramos Varanda ${ }^{1}$

Marina Minardi Nascimento ${ }^{1}$

José Eduardo Pompeu ${ }^{1}$

\begin{abstract}
Frailty can be defined as a biologic syndrome that results from decline in multiple systems leading to vulnerability, with poorer capacity of the organism to maintain homeostasis facing stressing events. Physical symptoms of frailty involve unintentional weight loss, decreased physical activity level, exhaustion, muscle weakness, impaired balance and gait. Together, these symptoms can compromise independence in activities of daily living. Physical Therapy can improve muscle strength, balance, gait and cardiorespiratory aptitude of frail elderly, but remains unclear what is the best intervention strategy: unimodal or multimodal interventions, with combined exercises. This systematic review aimed: (1) to compare the efficacy of unimodal and multimodal Physical Therapy interventions; (2) to analyze its effects on clinical outcomes and (3) to elucidate what is the best modality of exercise and its combination in order to improve physical symptoms and independence in frail elderly. Searches were conducted in PubMed, Medline, PEDro, Lilacs and Scielo databases. We obtained 2,579 studies and 17 were included in analysis. Studies analyzed the following clinical outcomes: mobility, muscle strength, balance, falls and fear of falling, quality of life and functional capacity. We conclude that both unimodal and multimodal interventions have potential to promote beneficial effects, but the analyzed studies have important methodological issues that negatively affect their quality. Although the beneficial effects of both interventions, the quality of studies analyzed does not allow us to generalize these effects. We suggest that new clinical trials should be conducted with a stronger methodology and according to current guidelines for randomized clinical trials.
\end{abstract}

\section{KEYWORDS}

Elderly; Frail Elderly; Gerontology; Physical Therapy Modalities; Rehabilitation.

\begin{abstract}
RESUMO
A fragilidade pode ser definida como uma síndrome biológica que resulta da diminuição da função de diversos sistemas, conduzindo à vulnerabilidade e pior capacidade do organismo para manter a homeostase frente a eventos estressores. As características físicas da fragilidade envolvem perda involuntária de peso, diminuição do nível de atividade física, cansaço, fraqueza muscular, diminuição do equilíbrio e marcha. Esses sintomas podem comprometer a independência nas atividades de vida diária. A fisioterapia pode melhorar a força muscular, equilíbrio, marcha e aptidão cardiorrespiratória de idosos frágeis, mas não está claro qual é a melhor estratégia: intervençôes unimodais ou multimodais, com exercícios combinados. Sendo assim, esta revisão sistemática objetivou: (1) comparar a eficácia de intervenções unimodais e multimodais; (2) analisar os efeitos sobre os resultados clínicos e (3) elucidar qual a melhor modalidade de exercício e suas combinações na melhora dos sintomas físicos e independência em idosos frágeis. Foram realizadas buscas nas bases de dados PubMed, Medline, Pedro, Lilacs e Scielo. Obtivemos 2.579 estudos e 17 foram incluídos na análise. Os estudos incluídos analisaram os desfechos mobilidade, força muscular, equilibrio, quedas e medo de cair, qualidade de vida e capacidade funcional. Concluimos que as intervenções unimodais e multimodais têm potencial para promover efeitos benéficos, mas os estudos analisados apresentaram falhas metodológicas que afetam a qualidade dos mesmos. Apesar dos benefícios de ambas as intervenções, a qualidade dos estudos não nos permite generalizar esses efeitos. Sugerimos que novos ensaios clínicos devam ser conduzidos, com metodologia de acordo com as recomendaçôes para ensaios clínicos randomizados.
\end{abstract}

Rev Bras Ativ Fís Saúde p. 458-466 DOI

http://dx.doi.org/10.12820/rbafs.v.20n5p458

'University of Sao Paulo, School of Medicine, Department of Speech, Physical Therapy and Occupational Therapy. Sao Paulo, Sao Paulo, Brazil. 


\section{INTRODUCTION}

Frailty can be defined as a biologic syndrome that results from decline in multiple systems leading to vulnerability, with poorer capacity of the organism to maintain homeostasis facing stressing events ${ }^{1,2}$. Its prevalence increases with age and is associated with negative health outcomes such as falls, disabilities and death ${ }^{1,2}$.

Due to difficulty in identifying frail older adults, Fried et al. ${ }^{1}$ proposed a standardized definition of frailty through five characteristics: unintentional weight loss, exhaustion, slowness, weakness and low physical activity level. Together, these symptoms can compromise independence in activities of daily living ${ }^{2}$.

Giné-Garriga et al. ${ }^{3}$, in a recent systematic review and meta-analysis, showed that exercise interventions promote positive effects in physical functions of frail elderly, although modality and characteristics of exercise vary widely on literature.

Many researchers have analyzed the effects of unimodal interventions, which are based on only one type of exercise, while others have studied association of exercise modalities ${ }^{3}$. The most common Physical Therapy interventions are based on resistance training ${ }^{4,5}$, aerobic exercises ${ }^{5}$ and balance exercises $^{4,5}$.

Remains unclear if unimodal or multimodal interventions show better effects in frailty, neither which is the best combination of exercises. This systematic review aimed: (1) to compare the efficacy of unimodal and multimodal Physical Therapy interventions, (2) to analyze its effects on clinical outcomes and (3) to elucidate what is the best modality of exercise and its combination in order to improve physical symptoms and independence in frail elderly. Our hypothesis is that both, unimodal and multimodal exercises, in different combinations, can promote positive effects on clinical outcomes of frail elderly, but due to multifactorial characteristic of frailty, combined exercises can be more effective than unimodal exercises.

\section{METHODS}

Searches were conducted in PubMed, Medline, PEDro, Lilacs and Scielo databases. The descriptors and Boolean operators were: [frail elderly OR idoso frágil] AND [rehabilitation OR reabilitação], [exercise OR exercício] [physical therapy OR fisioterapia], [resistance training OR treinamento de resistência].

Only randomized controlled trials were selected, published in the period from 2009 to 2014, in English or Portuguese languages and that analyzed the effects of unimodal or multimodal interventions in treatment of frailty.

Studies were firstly selected by title by two researchers. Abstracts of selected studies were read by the same two researchers and, in case of disagreements concerning inclusion, a third researcher participated in decision. After selection by abstracts, studies were fully read.

Quality of selected studies was analyzed using PEDro scale, which evaluates randomized clinical trials through eleven dichotomized questions (yes/ no) those score from zero to ten. Every answer "yes" is scored, from question two to eleven. Scores on PEDro scale were not considered as exclusion criteria and were used only to classify and describe the selected studies ${ }^{6}$. 


\section{RESULTS}

We obtained 2,579 studies, but after filtering according with its design, just 197 were randomized clinical trials. After reading the titles, we excluded 138 studies that did not assessed the effects of unimodal or multimodal interventions in treatment of frailty, resulting in 59 studies. After exclusion of duplicated studies, 17 were included in analysis, as shown in Figure 1.

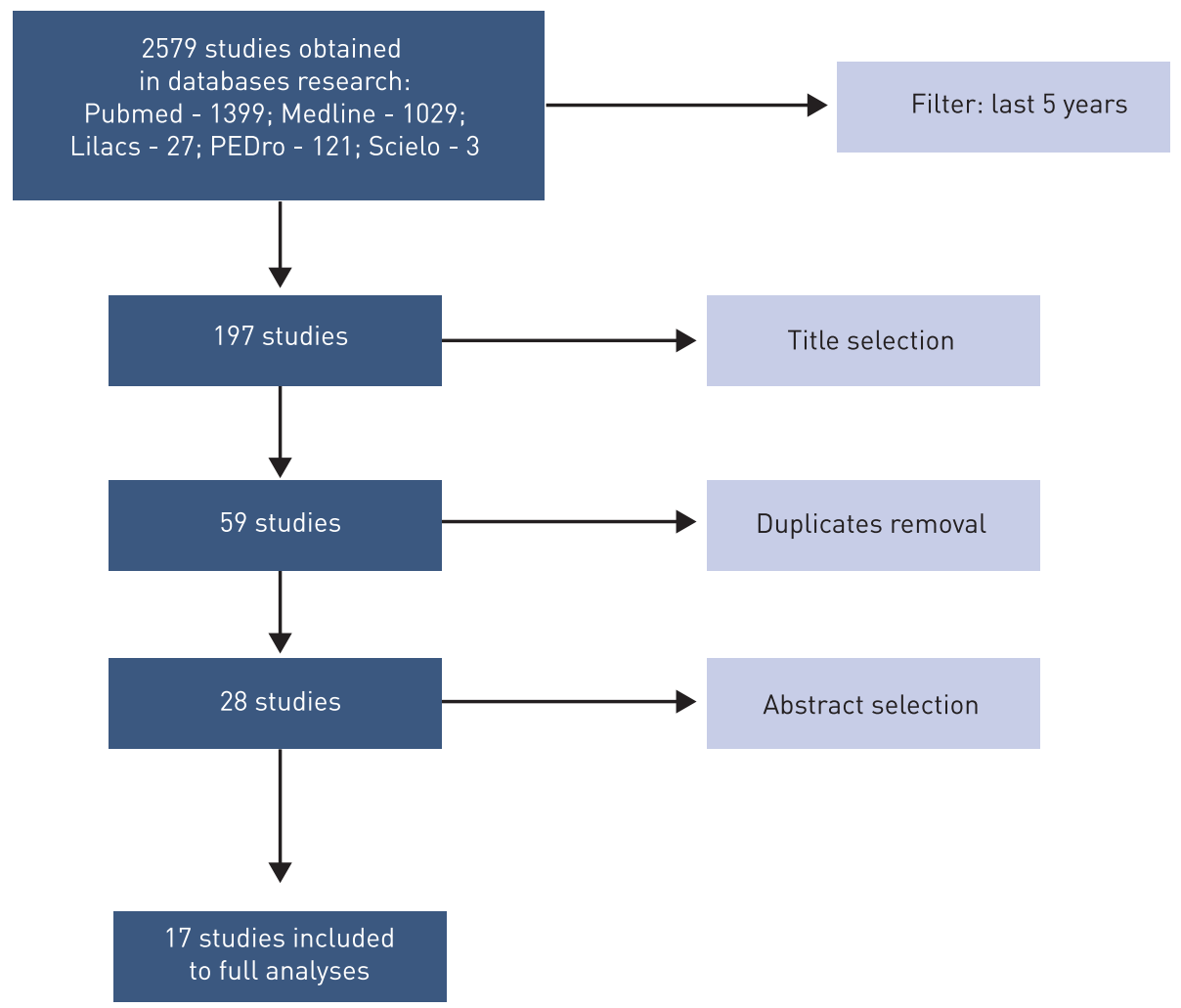

FIGURE 1 - Flow of research and selection of studies.

Selected studies analyzed the following clinical outcomes: mobility, muscle strength, balance, falls and fear of falling, quality of life and functional capacity.

Eleven studies ${ }^{7-16}$ analyzed mobility through the Timed Up \& Go test(TUG), walking speed test, Performance-Oriented Mobility Assessment (POMA), five meters walking test, ten meters walking test and six minute walking test.

Muscle strength was analyzed in ten studies ${ }^{7-14,17,18}$ through isokinetic dynamometer, manual dynamometer, grip strength, sit-to-stand five times, sitto-stand 30 seconds and sit-to-stand transfer muscle power.

Nine studies ${ }^{8,10,13-16,18-20}$ evaluated balance through Frailty and Injuries Cooperative Studies of Intervention Techniques (FICSIT-4), Short Physical Performance Battery (SPPB), Berg Balance Scale (BBS), baropodometry, tandem, semitandem and unipedal stance.

Falls were assessed in only one study ${ }^{14}$ through falls incidence inventory, while fear of falling was assessed in four ${ }^{21,23,24,28}$, using Falls Efficacy Scale International (FES-I) and Activities-specific Balance Confidence scale (ABC).

Quality of life was analyzed in four studies ${ }^{8,15,19,21}$ through Medical Outcomes Studies 12-item Short Form (SF-12) and EuroQol-5D (EQ-5D). 
Five studies ${ }^{10,13,17,19,22}$ analyzed functional capacity through Barthel index and Functional Independence Measure.

Table 1 shows the results of PEDro scale analysis concerning methodological quality of selected studies and table 2 shows the description of studies regarding interventions, sample size, outcomes and results.

TABLE 1 - PEDro scale scores.

\begin{tabular}{|c|c|c|c|c|c|c|c|c|c|c|c|c|}
\hline Authors/Year & 1 & 2 & 3 & 4 & 5 & 6 & 7 & 8 & 9 & 10 & 11 & TOTAL \\
\hline Cadore et al. ${ }^{14}$ & $Y$ & $Y$ & $Y$ & $Y$ & $\mathrm{~N}$ & $\mathrm{~N}$ & $\mathrm{~N}$ & $\mathrm{~N}$ & $\mathrm{Y}$ & $Y$ & $Y$ & 6 \\
\hline Cameron et al. ${ }^{19}$ & Y & $\mathrm{N}$ & $\mathrm{N}$ & Y & $\mathrm{N}$ & $\mathrm{N}$ & $\mathrm{N}$ & Y & Y & $Y$ & $Y$ & 5 \\
\hline Coelho et al. ${ }^{9}$ & Y & $Y$ & $\mathrm{~N}$ & Y & $\mathrm{N}$ & $\mathrm{N}$ & N & Y & Y & Y & Y & 6 \\
\hline Fairhall et al. ${ }^{24}$ & $Y$ & Y & $Y$ & $Y$ & $\mathrm{~N}$ & $\mathrm{~N}$ & $Y$ & Y & $Y$ & Y & Y & 8 \\
\hline Giné-Garriga et al. ${ }^{10}$ & $Y$ & $Y$ & $Y$ & $Y$ & $\mathrm{~N}$ & $\mathrm{~N}$ & $Y$ & $\mathrm{~N}$ & $Y$ & $Y$ & $Y$ & 7 \\
\hline Giné-Garriga et al. ${ }^{21}$ & $Y$ & $\mathrm{Y}$ & $Y$ & $Y$ & $\mathrm{~N}$ & $\mathrm{~N}$ & $Y$ & $\mathrm{~N}$ & $Y$ & $Y$ & $Y$ & 7 \\
\hline Gronstedt et al..$^{13}$ & $Y$ & $Y$ & $Y$ & $Y$ & $\mathrm{~N}$ & $Y$ & $Y$ & $\mathrm{~N}$ & $Y$ & $Y$ & Y & 8 \\
\hline Hagedorn et al. ${ }^{16}$ & $Y$ & Y & Y & Y & $\mathrm{N}$ & $\mathrm{N}$ & $\mathrm{N}$ & $\mathrm{N}$ & Y & Y & Y & 6 \\
\hline Langlois et al. ${ }^{12}$ & $Y$ & $Y$ & $\mathrm{~N}$ & $Y$ & $\mathrm{~N}$ & $\mathrm{~N}$ & N & N & $Y$ & $Y$ & Y & 5 \\
\hline Lustosa et al. ${ }^{7}$ & Y & Y & Y & Y & Y & Y & Y & $\mathrm{N}$ & Y & Y & $\mathrm{N}$ & 8 \\
\hline Molino-Lova et al. ${ }^{20}$ & $Y$ & Y & Y & $\mathrm{N}$ & $\mathrm{N}$ & $\mathrm{N}$ & $\mathrm{N}$ & $Y$ & $Y$ & $Y$ & Y & 6 \\
\hline Pollock et al. ${ }^{15}$ & $Y$ & $\mathrm{Y}$ & $Y$ & $Y$ & $\mathrm{~N}$ & $\mathrm{~N}$ & $Y$ & $\mathrm{~N}$ & $Y$ & $Y$ & Y & 7 \\
\hline Rydwik et al. ${ }^{22}$ & $Y$ & $\mathrm{~N}$ & $\mathrm{~N}$ & $Y$ & $\mathrm{~N}$ & $\mathrm{Y}$ & $\mathrm{N}$ & $\mathrm{N}$ & $\mathrm{Y}$ & $Y$ & $Y$ & 5 \\
\hline Sato et al. ${ }^{17}$ & $Y$ & Y & $Y$ & $Y$ & $Y$ & $\mathrm{~N}$ & $\mathrm{~N}$ & Y & $Y$ & Y & $\mathrm{N}$ & 7 \\
\hline Zak et al. ${ }^{11}$ & $Y$ & $Y$ & $Y$ & $Y$ & N & $\mathrm{N}$ & $Y$ & Y & $Y$ & $Y$ & Y & 8 \\
\hline Zech et al. ${ }^{18}$ & Y & $Y$ & Y & Y & N & $N$ & $\mathrm{~N}$ & Y & Y & Y & Y & 7 \\
\hline Zhang et al. ${ }^{8}$ & $Y$ & $Y$ & Y & Y & Y & $\mathrm{N}$ & N & Y & Y & Y & Y & 8 \\
\hline
\end{tabular}

Abbreviations: Y: yes; N: no.

TABLE 2 - Description of selected studies.

\begin{tabular}{|c|c|c|c|c|c|}
\hline Author & Intervention group & $\begin{array}{l}\text { Control } \\
\text { group }\end{array}$ & $N$ & Outcomes & Results \\
\hline $\begin{array}{l}\text { Cadore et } \\
\text { al. }{ }^{14}\end{array}$ & $\begin{array}{l}12 \mathrm{wks}, 2 \mathrm{x} / \mathrm{wk}, 40 \mathrm{~min} / \text { day; upper } \\
\text { and lower body resistance training, } \\
\text { balance and gait exercises, } \\
\text { functional exercises }\end{array}$ & $\begin{array}{l}12 \mathrm{wks}, 4 \times / \mathrm{wk}, 30 \\
\mathrm{~min} / \mathrm{day} ; \text { mobility } \\
\text { exercises }\end{array}$ & 32 & $\begin{array}{l}\text { Gait ability, dual- } \\
\text { task performance, } \\
\text { balance, LLMS, } \\
\text { ULMS, incidence } \\
\text { of falls, functional } \\
\text { status, muscle cross- } \\
\text { sectional area and } \\
\text { quality }\end{array}$ & $\begin{array}{l}\text { IG: increased gait speed, } \\
\text { decreased TUG, increased times } \\
\text { raised from a chair, improved } \\
\text { balance, lower deterioration } \\
\text { on Barthel Index, decreased } \\
\text { incidence of falls, better } \\
\text { performance on dual task, } \\
\text { increased ULMS/LLMS, increased } \\
\text { muscle cross-sectional area }\end{array}$ \\
\hline $\begin{array}{l}\text { Cameron } \\
\text { et al. }{ }^{19}\end{array}$ & $\begin{array}{l}12 \text { months; multifactorial and } \\
\text { interdisciplinary program targeted } \\
\text { to each individual's frailty criteria, } \\
\text { additional interventions based on } \\
\text { comprehensive geriatric evaluation }\end{array}$ & Usual care & 241 & $\begin{array}{l}\text { Frailty, mobility, } \\
\text { hospitalizations/ } \\
\text { admissions to nursing } \\
\text { care facilities, disability, } \\
\text { health-related QoL, } \\
\text { psychological status, } \\
\text { death }\end{array}$ & $\begin{array}{l}\text { Reduced frailty, improved } \\
\text { mobility, decreased mobility- } \\
\text { related disabilities. Benefits not } \\
\text { evidenced at follow-up }\end{array}$ \\
\hline $\begin{array}{l}\text { Coelho et } \\
\text { al. }{ }^{9}\end{array}$ & $\begin{array}{l}10 \text { wks, 3x/wk; progressive dynamics } \\
\text { resistance training (pre-frails) }\end{array}$ & $\begin{array}{l}10 \text { wks, } 3 \times / \text { wk; } \\
\text { progressive dynamic } \\
\text { resistance training } \\
\text { (non-frails) }\end{array}$ & 48 & $\begin{array}{l}\text { Neurotrophic } \\
\text { factors productions, } \\
\text { cognitive } \\
\text { performance, } \\
\text { depression, frailty } \\
\text { phenotype, muscle } \\
\text { strength, muscle } \\
\text { performance, } \\
\text { functional mobility }\end{array}$ & $\begin{array}{l}\text { Increased muscle strength, } \\
\text { plasma levels of BDNF } \\
\text { increased in both groups but } \\
\text { more in non-frails, functional } \\
\text { improvement }\end{array}$ \\
\hline
\end{tabular}




\begin{tabular}{|c|c|c|c|c|c|}
\hline Author & Intervention group & $\begin{array}{l}\text { Control } \\
\text { group }\end{array}$ & $\mathrm{N}$ & Outcomes & Results \\
\hline $\begin{array}{l}\text { Fairhall et } \\
\text { al. }{ }^{24}\end{array}$ & $\begin{array}{l}12 \text { months; multifactorial program } \\
\text { targeted to frailty, } 10 \text { sessions of } \\
\text { PT targeted to mobility, increase } \\
\text { physical activity and falls prevention }\end{array}$ & Usual care & 241 & $\begin{array}{l}\text { Mobility-related } \\
\text { disability, participation, } \\
\text { mobility performance, } \\
\text { mobility-related } \\
\text { participation, self- } \\
\text { report measure of } \\
\text { activity, adverse events }\end{array}$ & $\begin{array}{l}\text { Increased participation and } \\
\text { mobility, mobility-related } \\
\text { participation and gait speed }\end{array}$ \\
\hline $\begin{array}{l}\text { Giné- } \\
\text { Garriga et } \\
\text { al. }^{10}\end{array}$ & $\begin{array}{l}12 \text { wks, } 2 x / \text { wk; functional circuit } \\
\text { training (functional balance and } \\
\text { strength exercises) }\end{array}$ & $\begin{array}{l}12 \text { wks, once a week; } \\
\text { usual care + social } \\
\text { meetings }\end{array}$ & 51 & $\begin{array}{l}\text { Physical frailty } \\
\text { component, balance, } \\
\text { gait, physical function, } \\
\text { muscle strength }\end{array}$ & $\begin{array}{l}\text { Improves on physical } \\
\text { performance measures, reduced } \\
\text { physical frailty components }\end{array}$ \\
\hline $\begin{array}{l}\text { Giné- } \\
\text { Garriga et } \\
\text { al. }{ }^{21}\end{array}$ & $\begin{array}{l}12 \text { wks, } 2 x / \text { wk; functional circuit } \\
\text { training (functional balance and } \\
\text { strength exercises) }\end{array}$ & $\begin{array}{l}12 \text { wks, once a week; } \\
\text { usual care + social } \\
\text { meetings }\end{array}$ & 51 & Fear of falling, QoL & $\begin{array}{l}\text { Reduced fear of falling, } \\
\text { increased QoL }\end{array}$ \\
\hline $\begin{array}{l}\text { Gronstedt } \\
\text { et al. } .^{13}\end{array}$ & $\begin{array}{l}12 \text { wks; goal-oriented training } \\
\text { including transfers, walking, } \\
\text { balance, muscle strength and } \\
\text { endurance, outdoor walking, } \\
\text { gymnastics, personal care, social } \\
\text { activities }\end{array}$ & Usual care & 322 & $\begin{array}{l}\text { ADL, functional } \\
\text { balance, physical } \\
\text { activity level, } \\
\text { mobility, grip } \\
\text { strength, falls- } \\
\text { related self-efficacy }\end{array}$ & $\begin{array}{l}\text { Increased physical activity level, } \\
\text { balance, transfers, walking } \\
\text { speed, leg muscle strength }\end{array}$ \\
\hline $\begin{array}{l}\text { Hagedorn } \\
\text { et al. } .^{16}\end{array}$ & $\begin{array}{l}12 \text { wks, } 2 x / \text { wk; muscle strength } \\
\text { training, physical fitness training, } \\
\text { home exercises, computer feedback } \\
\text { balance training }\end{array}$ & $\begin{array}{l}12 \text { wks, } 2 x / \text { wk; } \\
\text { muscle strength } \\
\text { training, physical } \\
\text { fitness training, } \\
\text { home exercises, } \\
\text { traditional balance } \\
\text { training }\end{array}$ & 27 & $\begin{array}{l}\text { LLMS, ULMS, } \\
\text { lower-body strength, } \\
\text { mobility, aerobic } \\
\text { endurance, balance, } \\
\text { gait, fear of falling }\end{array}$ & $\begin{array}{l}\text { Both groups: increased } \\
\text { muscle strength, decreased } \\
\text { fear of falling; CG: increased } \\
\text { lower-body muscle strength, } \\
\text { increased balance; IG: increased } \\
\text { endurance }\end{array}$ \\
\hline $\begin{array}{l}\text { Langlois } \\
\text { et al. }{ }^{12}\end{array}$ & $\begin{array}{l}12 \text { wks, } 3 x / \text { wk; stretching and } \\
\text { balance exercises, aerobic exercises, } \\
\text { strength training }\end{array}$ & Usual care & 83 & $\begin{array}{l}\text { Physical capacity, } \\
\text { cognition, QoL }\end{array}$ & $\begin{array}{l}\text { IG improved in functional } \\
\text { capacity, physical endurance, } \\
\text { cognition and QoL }\end{array}$ \\
\hline $\begin{array}{l}\text { Lustosa et } \\
\text { al. }{ }^{7}\end{array}$ & $\begin{array}{l}10 \text { wks, } 3 x / \text { wk; lower limbs strength } \\
\text { training with } 75 \% \text { of } 1 \text { RM, } 3 \text { series of } \\
8 \text { repetitions }\end{array}$ & Usual care & 32 & $\begin{array}{l}\text { Functional } \\
\text { performance, knee } \\
\text { extensors muscle } \\
\text { strength }\end{array}$ & $\begin{array}{l}\text { Training provided increased } \\
\text { muscle power and functional } \\
\text { performance, with no } \\
\text { differences in muscle strength }\end{array}$ \\
\hline $\begin{array}{l}\text { Molino- } \\
\text { Lova et } \\
\text { al. }^{20}\end{array}$ & $\begin{array}{l}12 \text { months; aerobic exercises + } \\
\text { home-based } 3 x / \text { wk exercises for } \\
\text { muscle strengthening, flexibility, } \\
\text { balance and coordination }\end{array}$ & Aerobic exercises & 140 & Functional capacity & $\begin{array}{l}\text { IG presented greater } \\
\text { improvements in functional } \\
\text { capacity }\end{array}$ \\
\hline $\begin{array}{l}\text { Pollock et } \\
\text { al. }{ }^{15}\end{array}$ & $\begin{array}{l}8 \text { wks, } 3 x / \text { wk; whole-body vibration } \\
+ \text { exercises targeting muscle } \\
\text { strengthening, balance and } \\
\text { functional mobility }\end{array}$ & $\begin{array}{l}\text { Exercises } \\
\text { targeting muscle } \\
\text { strengthening, } \\
\text { balance and } \\
\text { functional mobility }\end{array}$ & 77 & $\begin{array}{l}\text { Functional mobility, } \\
\text { sarcopenia, } \\
\text { functional balance, } \\
\text { fear of falling, } \\
\text { health-related QoL }\end{array}$ & $\begin{array}{l}\text { IG presented greater } \\
\text { improvements in functional } \\
\text { mobility }\end{array}$ \\
\hline $\begin{array}{l}\text { Rydwik et } \\
\text { al. }{ }^{22}\end{array}$ & $\begin{array}{l}\text { 2x/wk, } 12 \text { wks; I: specific physical } \\
\text { training + general diet advice; II: } \\
\text { specific physical training + specific } \\
\text { diet counseling + group-session } \\
\text { training; III: specific diet counseling } \\
+ \text { group-session training + general } \\
\text { physical training advice }\end{array}$ & $\begin{array}{l}\text { General physical } \\
\text { training advice }+ \\
\text { general diet advice }\end{array}$ & 96 & $\begin{array}{l}\text { Physical activity level } \\
\text { and ADL }\end{array}$ & $\begin{array}{l}\text { Groups I and II increased } \\
\text { physical activity levels; no } \\
\text { differences in any group for ADL }\end{array}$ \\
\hline $\begin{array}{l}\text { Sato, } \\
\text { Kaneda, } \\
\text { Nonura }^{17}\end{array}$ & $\begin{array}{l}2 \text { yrs - } 2 x / \text { wk; hydrotherapy with } \\
\text { ADL training + stretching + muscle } \\
\text { strength training + relaxation }\end{array}$ & $\begin{array}{l}2 \text { yrs }-1 x / \text { wk; } \\
\text { hydrotherapy with } \\
\text { ADL training + } \\
\text { stretching + muscle } \\
\text { strength training + } \\
\text { relaxation }\end{array}$ & 22 & $\begin{array}{l}\text { ADL abilities and } \\
\text { LLMS }\end{array}$ & $\begin{array}{l}\text { Both groups improved in ADL } \\
\text { abilities; twice weekly group } \\
\text { improved LLMS }\end{array}$ \\
\hline Zak et al. ${ }^{11}$ & $\begin{array}{l}\text { 5x/wk, } 7 \text { wks; I: progressive } \\
\text { resistance exercises + } \\
\text { nutritional supplementation; III: } \\
\text { standard exercises + nutritional } \\
\text { supplementation }\end{array}$ & $\begin{array}{l}\text { II: progressive } \\
\text { resistance exercises } \\
\text { + placebo nutritional } \\
\text { supplementation; IV: } \\
\text { standard exercises + } \\
\text { placebo nutritional } \\
\text { supplementation }\end{array}$ & 91 & $\begin{array}{l}\text { Functional } \\
\text { performance } \\
\text { capability, muscle } \\
\text { strength, nutritional } \\
\text { aspects }\end{array}$ & $\begin{array}{l}\text { Groups I and II improved muscle } \\
\text { strength, group III improved } \\
\text { mobility and body weight }\end{array}$ \\
\hline
\end{tabular}




\begin{tabular}{|c|c|c|c|c|c|}
\hline Author & Intervention group & $\begin{array}{l}\text { Control } \\
\text { group }\end{array}$ & $\mathrm{N}$ & Outcomes & Results \\
\hline $\begin{array}{l}\text { Zech et } \\
\text { al. }{ }^{18}\end{array}$ & $\begin{array}{l}2 x / \text { wk, } 12 \text { wks; one group performed } \\
\text { muscle strength training and one } \\
\text { group performed muscle power } \\
\text { training }\end{array}$ & $\begin{array}{l}\text { Maintained usual } \\
\text { physical activity level }\end{array}$ & 69 & $\begin{array}{l}\text { Physical } \\
\text { performance, } \\
\text { muscular power of } \\
\text { LL, self-reported } \\
\text { function of LL and } \\
\text { upper extremities }\end{array}$ & $\begin{array}{l}\text { Both intervention groups } \\
\text { increased in physical function, } \\
\text { but gains were not maintained } \\
\text { after detraining period }\end{array}$ \\
\hline $\begin{array}{l}\text { Zhang et } \\
\text { al. }{ }^{8}\end{array}$ & $\begin{array}{l}8 \text { wks - 3-5x/wk; Whole-body } \\
\text { vibration }\end{array}$ & $\begin{array}{l}8 \text { wks; usual care } \\
+ \text { PT + routine } \\
\text { exercises }\end{array}$ & 44 & $\begin{array}{l}\text { Mobility, LLMS, } \\
\text { balance, balance } \\
\text { confidence, postural } \\
\text { control, general } \\
\text { health status, } \\
\text { subjective experience } \\
\text { and training } \\
\text { preference }\end{array}$ & $\begin{array}{l}\text { Both groups showed } \\
\text { improvements of all measures } \\
\text { at } 04 \text { and } 08 \text { wks; more } \\
\text { pronounced improvements in } \\
\text { intervention group regarding } \\
\text { mobility, LL, balance confidence } \\
\text { and general health status }\end{array}$ \\
\hline
\end{tabular}

Abbreviations: wks: weeks; wk: week; min: minutes; LLMS: lower limbs muscle strength; ULMS: upper limbs muscle strength; IG: intervention group; CG: control group; QoL: quality of life; BDNF: Brain-derived neurotrophic factor; PT: Physical Therapy; ADL: activities of daily living; RM: repetition maximum; yr: year; LL: lower limbs.

\section{DISCUSSION}

Regarding quality of studies assessed through PEDro scale, most studies were scored as 7 or 8 (10 of 17 studies) and the other studies were scored between 5 and 6 (7 of 10 studies), which doesn't allow us to infer good methodological quality. However, 2 studies did not describe the method of randomization, 4 studies did not perform a blind distribution of participants between groups, 1 study did not have homogenous samples at the beginning of the study, 14 studies had problems concerning blinding in treatments, both to participants and therapists, 9 studies had more than $25 \%$ of drop out and 2 studies did not have measures of precision or variability of the results. These issues decreases the quality of the studies and can compromise the confidence in their results. Specifically about blindness of interventions, it is difficult that participants and therapists do not know what interventions they are participant but, it is possible and recommended the blindness of the assessor. Drop out higher than $25 \%$ can cause problems with statistical analysis and, at the same time, can indicate that the interventions of the studies had problems of adherence of participants.

Despite diversity among modalities of exercises observed in multimodal interventions, around $80 \%$ of studies included muscle strength training, balance training and functional activities in their protocols. Diversity was also observed regarding duration and frequency of exercises, but most protocols presented from 10 to 12 weeks of duration, 2 times per week and from 30 minutes to one hour. Apart the variation among exercise modalities, protocols varied regarding other interventions, like nutritional support ${ }^{11,22}$, social activities and personal care ${ }^{10,13,21}$. The high diversity among the variables of the studies makes impossible the comparison among them and we cannot affirm what is the better combination of exercises.

Incidence of falls was assessed only by one study ${ }^{14}$, which showed that multimodal interventions were more effective in reducing the incidence of falls when compared to unimodal intervention. Therefore, more studies should be done in order to elucidate what is the better combination of exercises to improve balance. About fear of falling, none of studies compared unimodal with 
multimodal interventions but both modalities showed beneficial effects. Only two studies ${ }^{13,16}$ did not find these effects on fear of falling. Considering the multifactorial characteristics of falls, multimodal interventions that treat many risk factors as possible, would be more effective than unimodal interventions. However, it is necessary a study that aims to compare unimodal with multimodal interventions regarding fear for fall in order to confirm our speculation.

Regarding quality of life, protocols and comparison between groups were quite different among studies ${ }^{8,15,19,21}$. Except for one study ${ }^{19}$, both unimodal and multimodal interventions showed positive effects on quality of life. We speculate that these positive effects are related to the fact that quality of life can be affected by many variables, such as mental and physical status, the main outcome of most studies. Based on the multifactorial characteristic of quality of life, probably, interventions that are more comprehensive can produce higher effects. However, this speculation needs to be evaluated with a study specifically designed for this aim.

When analyzing functional capacity results vary among studies ${ }^{10,13,17,19,22}$, being difficult to establish the possible effects of interventions. We observed differences not only among interventions protocols, but also among assessments tools. Therefore, the effects of unimodal and multimodal exercises should be investigated.

Muscle strength seems to ameliorate through both unimodal and multimodal interventions ${ }^{7-14,17,18}$. Only one study did not show positive effects in muscle strength ${ }^{12}$. Improves in muscle strength are often obtained through exercise programs specifically aimed to that ${ }^{23}$ and, in frailty, this physical component is related to gains in functional abilities ${ }^{2}$.

Regarding balance, all studies ${ }^{7-15,17-22,24}$ except for one ${ }^{16}$, showed positive effects through both unimodal and multimodal interventions. Even considering the variety of interventions across studies, balance improvement was observed and may not only positively affect frail elderly people, but also prevent falls in this population. However, new longitudinal studies are necessary in order to assess the effects of balance exercises in the incidence of falls in frail elderly.

Mobility was improved through both unimodal and multimodal interventions $^{7-12,15,16,24}$ and this finding are of most importance to clinical practice, since muscle strengthening, balance and mobility training are widely used in treatment of frailty and fall prevention.

According to the physical therapy interventions analyzed in this review, we conclude that both unimodal and multimodal interventions have potential to promote beneficial effects, but the analyzed studies have important methodological issues that negatively affect the quality of the studies. The results of the included studies suggest that unimodal interventions can promote changes in specific therapeutic goals, while multimodal interventions can produce most comprehensive effects, possibly optimizing rehabilitation period to more goals achievements in reduced time. However, due the methodological issues of the studies, the evidences of this affirmation should be better investigated. All clinical outcomes analyzed by studies showed improvements with unimodal and multimodal exercises. These results suggest that the best intervention program would be a combination of specific exercises according to the more pronounced deficiencies of frail elderly. Although we observed beneficial effects of both interventions, the quality of studies analyzed does 
not allow us to generalize these effects. We suggest that new clinical trials should be conducted with a stronger methodology and according to current guidelines for randomized clinical trials.

\section{Acknowledgments}

No financial support.

\section{Disclosure statement}

No potential conflicts of interest were disclosed.

\section{Authors' contributions}

Simões, M.S.M.P.: contributed to conception and design, interpretation of data, writing and revising the text and final approval.

Moura, P.A., Varanda, R., Nascimento, M.M.: contributed to conception and design, acquisition, analysis and interpretation of data, revising the text and final approval.

Pompeu, J.E.: contributed to conception and design, interpretation of data, revising the text and final approval.

\section{REFERENCES}

1. Fried LP, Tangen CM, Walston J, Newman AB, Hirsch C, Gottdiener J, et al. Frailty in older adults: evidence for a phenotype. J Gerontol A Biol Sci Med Sci. 2001;56(3):M146-56.

2. Clegg A, Young J, Iliffe S, Rikkert MO, Rockwood K. Frailty in elderly people. Lancet. 2013;381(9868):752-62.

3. Giné-Garriga M, Roqué-Fíguls M, Coll-Planas L, Sitjà-Rabert M, Salvà A. Physical exercise interventions for improving performance-based measures of physical function in community-dwelling, frail older adults: a systematic review and meta-analysis. Arch Phys Med Rehabil. 2014;95(4):753-69.e3.

4. Macedo C, Gazzola JM, Najas M. Síndrome da fragilidade no idoso: importância da fisioterapia. Arq Bras Ciên Saúde. 2008;33(3):8.

5. Arantes P, Alencar M, Dias R, Dias J, Pereira L. Physical therapy treatment on frailty syndrome: systematic review. Rev Bras Fisioter. 2009;13(5):365-75.

6. Costa CML. Tradução e adaptação da PEDro Scale para a cultura portuguesa: um instrumento de avaliação de ensaios clínicos em Fisioterapia. Lisboa: Universidade Técnica de Lisboa; 2011.

7. Lustosa LP, Silva JP, Coelho FM, Pereira DS, Parentoni AN, Pereira LS. Impact of resistance exercise program on functional capacity and muscular strength of knee extensor in pre-frail community-dwelling older women: a randomized crossover trial. Rev Bras Fisioter. 2011;15(4):318-24.

8. Zhang L, Weng C, Liu M, Wang Q, Liu L, He Y. Effect of whole-body vibration exercise on mobility, balance ability and general health status in frail elderly patients: a pilot randomized controlled trial. Clin Rehabil. 2014;28(1):59-68.

9. Coelho FM, Pereira DS, Lustosa LP, Silva JP, Dias JM, Dias RC, et al. Physical therapy intervention (PTI) increases plasma brain-derived neurotrophic factor (BDNF) levels in non-frail and pre-frail elderly women. Arch Gerontol Geriatr. 2012;54(3):415-20.

10. Giné-Garriga M, Guerra M, Pagès E, Manini TM, Jiménez R, Unnithan VB. The effect of functional circuit training on physical frailty in frail older adults: a randomized controlled trial. J Aging Phys Act. 2010;18(4):401-24.

11. Zak M, Swine C, Grodzicki T. Combined effects of functionally-oriented exercise regimens and nutritional supplementation on both the institutionalised and free-living frail elderly (double-blind, randomised clinical trial). BMC Public Health. 2009;9:39.

12. Langlois F, Vu TT, Chassé K, Dupuis G, Kergoat MJ, Bherer L. Benefits of physical exercise training on cognition and quality of life in frail older adults. J Gerontol B Psychol Sci Soc Sci. 2013;68(3):400-4. 
13. Grönstedt H, Frändin K, Bergland A, Helbostad JL, Granbo R, Puggaard L, et al. Effects of individually tailored physical and daily activities in nursing home residents on activities of daily living, physical performance and physical activity level: a randomized controlled trial. Gerontology. 2013;59(3):220-9.

14. Cadore EL, Casas-Herrero A, Zambom-Ferraresi F, Idoate F, Millor N, Gómez M, et al. Multicomponent exercises including muscle power training enhance muscle mass, power output, and functional outcomes in institutionalized frail nonagenarians. Age (Dordr). 2014;36(2):773-85.

15. Pollock RD, Martin FC, Newham DJ. Whole-body vibration in addition to strength and balance exercise for falls-related functional mobility of frail older adults: a singleblind randomized controlled trial. Clin Rehabil. 2012;26(10):915-23.

16. Hagedorn DK, Holm E. Effects of traditional physical training and visual computer feedback training in frail elderly patients. A randomized intervention study. Eur J Phys Rehabil Med. 2010;46(2):159-68.

17. Sato D, Kaneda K, Wakabayashi H, Nomura T. Comparison of 2-year effects of once and twice weekly water exercise on activities of daily living ability of community dwelling frail elderly. Arch Gerontol Geriatr. 2009;49(1):123-8.

18. Zech A, Drey M, Freiberger E, Hentschke C, Bauer JM, Sieber CC, et al. Residual effects of muscle strength and muscle power training and detraining on physical function in community-dwelling prefrail older adults: a randomized controlled trial. BMC Geriatr. 2012;12:68.

19. Cameron ID, Fairhall N, Langron C, Lockwood K, Monaghan N, Aggar C, et al. A multifactorial interdisciplinary intervention reduces frailty in older people: randomized trial. BMC Med. 2013;11:65.

20. Molino-Lova R, Pasquini G, Vannetti F, Paperini A, Forconi T, Polcaro P, et al. Effects of a structured physical activity intervention on measures of physical performance in frail elderly patients after cardiac rehabilitation: a pilot study with 1-year follow-up. Intern Emerg Med. 2013;8(7):581-9.

21. Giné-Garriga M, Guerra M, Unnithan VB. The effect of functional circuit training on self-reported fear of falling and health status in a group of physically frail older individuals: a randomized controlled trial. Aging Clin Exp Res. 2013;25(3):329-36.

22. Rydwik E, Frändin K, Akner G. Effects of a physical training and nutritional intervention program in frail elderly people regarding habitual physical activity level and activities of daily living--a randomized controlled pilot study. Arch Gerontol Geriatr. 2010;51(3):283-9.

23. Ciolac EG, Garcez-Leme LE, Greve JM. Resistance exercise intensity progression in older men. Int J Sports Med. 2010;31(6):433-8.

24. Fairhall N, Sherrington C, Kurrle SE, Lord SR, Lockwood K, Cameron ID. Effect of a multifactorial interdisciplinary intervention on mobility-related disability in frail older people: randomised controlled trial. BMC Med. 2012;10:120.

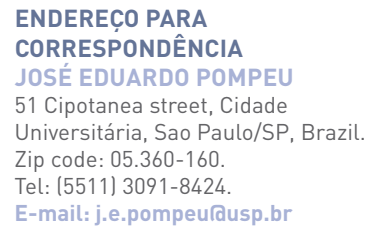

